\title{
BMJ Open Are hospital services for self-harm getting better? An observational study examining management, service provision and temporal trends in England
}

\author{
J Cooper, ${ }^{1}$ S Steeg, ${ }^{1}$ O Bennewith, ${ }^{2}$ M Lowe, ${ }^{1}$ D Gunnell, ${ }^{2}$ A House, ${ }^{3}$ K Hawton, ${ }^{4}$ \\ N Kapur ${ }^{1}$
}

To cite: Cooper J, Steeg S, Bennewith 0, et al. Are hospital services for self-harm getting better? An observational study examining management, service provision and temporal trends in England. BMJ Open 2013;3: e003444. doi:10.1136/ bmjopen-2013-003444

- Prepublication history and additional material for this paper is available online. To view these files please visit the journal online (http://dx.doi.org/ 10.1136/bmjopen-2013003444).

Received 19 June 2013 Revised 4 September 2013 Accepted 9 October 2013

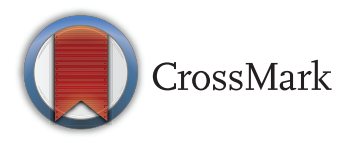

${ }^{1}$ Centre for Suicide Prevention, University of Manchester, Manchester, UK ${ }^{2}$ School of Social and Community Medicine, University of Bristol, UK ${ }^{3}$ Academic Unit of Psychiatry and Behavioural Sciences, Leeds, UK

${ }^{4}$ Centre for Suicide Research, Department of Psychiatry, University of Oxford, UK

Correspondence to Dr Jayne Cooper; jayne.cooper@manchester. ac.uk

\section{ABSTRACT}

Objectives: To describe the characteristics and management of individuals attending hospital with self-harm and assess changes in management and service quality since an earlier study in 2001, a period in which national guidance has been available.

Design: Observational study.

Setting: A stratified random sample of 32 hospitals in England, UK.

Participants:: 6442 individuals presenting with 7689 episodes of self-harm during a 3-month audit period between 2010 and 2011.

Outcome: Self-harm episodes, key aspects of individual management relating to psychosocial assessment and follow-up, and a 21-item measure of service quality.

Results: Overall, $56 \%(3583 / 6442)$ of individuals were women and $51 \%(3274 / 6442)$ were aged under 35 years. Hospitals varied markedly in their management. The proportion of episodes that received a psychosocial assessment by a mental health professional ranged from $22 \%$ to $88 \%$ (median $58 \%$, IQR 48-70\%); the proportion of episodes resulting in admission to general hospitals varied from $22 \%$ to $85 \%$ (median 54\%, IQR $41-63 \%$ ); a referral for specialist mental health follow-up was made in $11-64 \%$ of episodes (median 28\%, IQR 22-38\%); a referral to non-statutory services was made in 4-62\% of episodes (median 15\%, IQR 8-23\%); 0-21\% of episodes resulted in psychiatric admission (median $7 \%$, QR 4-12\%). The specialist assessment rate varied by method of harm; the median rate for self-cutting was $45 \%$ (IQR $28-63 \%$ ) vs $58 \%$ (IQR $48-73 \%$ ) for self-poisoning. Compared with the 2001 study, there was little difference in the proportion of episodes receiving specialist assessment; there was a significant increase in general hospital admission but a decrease in referrals for specialist mental health follow-up. However, scores on the service quality scale had increased from a median of 11.5-14.5 (a $26 \%$ increase).

Conclusions: Services for the hospital management of self-harm remain variable despite national guidelines and policy initiatives. We found no evidence for increasing levels of assessment over time but markers of service quality may have improved.

\section{Strengths and limitations of this study}

- This was a large study of hospital attendances at emergency departments following self-harm using recent data at the individual and episode levels from multiple sites in England.

- For comparison purposes, we were able to include 31 of the original 32 sites from an earlier study carried out in 2001/2002.

- We only collected data on self-harm attendances at hospitals and did not record episodes that did not come to medical attention.

- Data sources were based on clinical records and some of our findings may therefore underestimate the true prevalence of particular characteristics or associated factors.

This paper forms part of the study 'Variations in selfharm service delivery: an observational study examining outcomes and temporal trends'. The National Institute for Health Research Clinical Research Network (NIHR CRN) Portfolio database registration number: HOMASH 2 (7333). The NIHR Coordinated System for gaining NHS Permission (CSP) registration number: 23226.

\section{INTRODUCTION}

Self-harm is a major cause of presentation to hospitals and is linked to an elevated risk of early death. ${ }^{1}$ Hospital services for self-harm in the UK over the past four decades have been characterised by variability of service provision $^{2}$ and contrasting patient experiences of care. ${ }^{3}{ }^{4}$ During the 1970 s, wide variation in the management of patients with self-harm was found in 10 psychiatric teams in one English city. ${ }^{5}$ Twenty years later, a twofold difference was seen in the proportion of patients receiving a psychosocial assessment following self-harm in four hospitals in the north-west of England. ${ }^{6}$ The most comprehensive study of the management of 
self-harm until now, conducted in 2001/2002 in 32 hospitals in England, found a 2-fold variation across hospitals in the levels of psychosocial assessment, a 4-fold variation in general hospital admission, a 10-fold variation in psychiatric hospital admission and striking differences in the organisation and provision of services for patients with self-harm. ${ }^{7}$ Subsequent to this, there have been several policy documents giving guidance on appropriate service structures and the hospital management of self-harm.

In 2004, two sets of clinical guidelines on the management of self-harm were published which included the recommendation that every patient presenting to hospital with self-harm should receive a psychosocial assessment before discharge from hospital. ${ }^{8}{ }^{9}$ In addition, the Royal College of Psychiatrists initiated the Better Services for People who Self-harm Project, ${ }^{10}$ an audit-based quality improvement project involving surveys of service users' experiences, staff attitudes and training and care pathways. The two sets of guidelines and the Better Services Project might be expected to reduce the variability of services and improve the quality of care for self-harm patients.

In the current study, we aimed to (1) describe the characteristics and hospital management of self-harm across the same sample of 32 hospitals that took part in our earlier study, (2) compare our findings with the results from the earlier study in order to explore whether the service variability had decreased and service quality had improved over a 10-year period.

\section{METHODS}

\section{Setting and sample}

A random sample of 32 hospitals was identified in our earlier investigation. ${ }^{7}$ The original sample was stratified so that four hospitals were selected within each of the eight former Health Regions in England. Hospitals with no emergency department (ED) on site were not included. Thirty-one of the original 32 hospitals agreed to take part in the current study. The one hospital that declined to participate was replaced by an alternative randomly selected hospital from within the same stratum, as identified in the earlier study. Hospitals provided data on episodes of self-harm presenting to the ED (for the service audits) and on the structure of selfharm services (the service interviews).

\section{Data collection}

Descriptive study

Descriptive data were collected and recorded locally on site by clinical staff or Clinical Studies Officers (employed by the Trusts or national research networks), with the central research team overseeing the set-up and administration of the data collection process. Guidelines for inclusion/exclusion criteria of individual items of data were provided to ensure data consistency between centres, with the research team answering specific queries. For each audit, data were collected on all episodes of self-harm in those aged 18 and over occurring during a 3-month period. Service configurations for young people were likely to be markedly different from adult services and therefore those under 18 were excluded from this study. The exact time period varied between centres but all audits took place between May 2010 and June 2011.

Episodes of self-harm were identified from ED records using relevant search terms from our previous study ${ }^{7}$ and from current self-harm monitoring systems in England. ${ }^{11}{ }^{12}$ The individual medical records of possible cases were examined to confirm case inclusion. As in the previous study, self-harm was defined as "a deliberate non-fatal act whether physical, drug over-dosage or poisoning, done in the knowledge that it was potentially harmful and in the case of drug overdose that the amount taken was excessive." 13 The robustness of this methodology was tested in each hospital in a pilot data collection exercise against all presentations for a brief period (1-2 weeks) and/or against lists of presentations compiled by mental health teams to identify missed cases. Search terms were adjusted accordingly to maximise case ascertainment. All data were anonymised at source (at the participating hospital) before being sent to the research team. A named person within the trust held the key to enable subsequent patient attendances/ episodes to be identified.

Individual-level data were collected using a one page data collection sheet (see online supplementary appendix 1), which included demographic and clinical data, method of harm and the patient's recent contact with specialist mental health services. Details of in-hospital management were also recorded, specifically whether the individual received a psychosocial assessment (defined as 'an interview carried out by a member of mental health staff who has been trained in the process, is usually of about $30 \mathrm{~min}$ duration, and covers the assessment of factors such as the causes and degree of suicidal intent, current mental state and level of social support, psychiatric history, personal and social problems, future risk and need for follow-up, ${ }^{13}$ ), whether they were admitted to a psychiatric or medical bed and whether they were referred for psychiatric follow-up. Data were collected from acute hospital and mental health medical records systems.

\section{Service interviews}

A key mental health and an ED clinician involved in the provision of self-harm services, identified by the Local Collaborator at each Trust, were interviewed on the telephone or in person about current service structures. Using the staff responses, hospitals were then rated on a measure of service quality developed as part of our previous study $^{7}$ (see online supplementary appendix 2:21 Items of Service Quality) and based on the Royal College of Psychiatrists Guidelines for the general hospital management of self-harm. ${ }^{9}$ These included the 
presence of a psychiatric liaison team within the ED, with appropriate support, training and supervision available for ED clinicians and psychiatric staff, regular multidisciplinary management meetings, contact arrangements with primary care and the existence of formal links with non-statutory services. Twenty-one items were scored ' 1 ' or ' 0 ' depending on the presence or absence of a particular aspect of the service. Consistency of coding was achieved by meetings and regular correspondence between key researchers from the earlier and current studies. For two items (supervision arrangements for mental health staff who undertake psychosocial assessments and emergency attendance by a mental health worker available to the hospital ED within $1 \mathrm{~h}$ ) where such a strict categorisation was not possible, scores of ' 0 ', ' 0.5 ' or ' 1 ' were given in consultation with the research team. Therefore, hospitals could be potentially scored up to 21 on the Service Scale.

A summary of the methodology used in the present study and differences and similarities with our previous study is shown in online supplementary appendix 3 .

\section{Analysis}

Analysis of the descriptive data was carried out both at the individual patient level and at the episode level. The characteristics of the cohort were examined based on each individual's first hospital presentation within the study period, the 'index' episode. The key aspects of clinical management were then measured using all episodes of selfharm (including any repeat presentations by the same individual during the data collection period) in the 31 hospitals that were included in both studies. This approach allowed us to make direct comparisons with the earlier study, where individuals were not identified.

Aggregated hospital-level data were used to compare the Service Scale scores of the hospitals and to examine the changes in total Service Scale score and levels of hospital management over time. Spearman's rank correlation coefficient was used to measure associations between levels of hospital key management (using categories as set out in table 2) and total service score. Differences in scores between the two time periods were tested using the matched-pairs signed-rank test. Analyses were conducted using Stata V.11 ${ }^{14}$ and SPSS V.19. ${ }^{15}$

\section{Local and ethical approval}

This study received ethical approval from Tameside and Glossop National Health Service (NHS) Research Ethics Committee in August 2009. The data collection process at each site was classified as a local audit and therefore patient consent was not required. Local approval was sought to carry out the study through the Research and Development departments at each participating NHS Trust. As part of this process, we approached potential local collaborators at each Trust commonly through the assistance of national research networks (eg, Mental Health Research Network).

\section{RESULTS}

\section{Characteristics of individuals}

A total of 6442 individuals presented with 7689 episodes of self-harm at the 32 hospitals during the 3-month data collection period. Overall, 56\% (3583) of individuals were women and $51 \%$ (3274) were aged under 35 years (age range 18-94; median age 34; IQR 24-45). Information on ethnicity was not widely available for seven of the hospitals. Data were $85 \%$ complete in the remaining hospitals (4333); 93\% (4017) of individuals were white, $3 \%$ (124) South Asian, 2\% (78) black and $3 \%$ (114) were from other ethnic groups.

The main method of self-harm was known in $99.7 \%$ (6424/6442) of index episodes: self-poisoning with drugs in $79 \%$ (5073) of individuals, self-poisoning (other, eg, bleach, antifreeze and batteries) in 2\% (102), self-cutting in 14\% (890) and other methods of harm (including burning, attempted hanging and jumping) in $6 \%$ (359). A more detailed consideration of the methods used is beyond the scope of this paper and will be reported later. Data completeness was at least $90 \%$ for all other variables. Alcohol was taken within $6 \mathrm{~h}$ of the self-harm act in 53\% (3111/5828) of cases and recreational drugs in $7 \%(385 / 5828)$; previous self-harm had occurred in $51 \%(3173 / 6237)$ of individuals; patients were receiving psychiatric treatment at the time of their index self-harm episode in 32\% (1982/6181) of cases; and $10 \%(636 / 6269)$ had been inpatients in a psychiatric ward in the 12 months prior to the self-harm presentation.

\section{Specialist assessment (all episodes)}

A psychosocial assessment by a mental health specialist took place in $57 \%$ of all presentations. Seventy-six per cent $(3109 / 4075)$ of assessments were carried out by a mental health nurse (including mental health liaison nurses and those from specialist self-harm teams and crisis teams), 20\% (799) by a psychiatrist (any grade) and $4 \%$ (167) by another mental health professional (such as a social worker). The median number of hours between time of hospital presentation and time of assessment was 11 (IQR 5-21). For those not admitted to a medical bed, the median time to assessment was $5 \mathrm{~h}$ (IQR 3-9) compared with $14 \mathrm{~h}$ (IQR 8-25) for those admitted. Episodes where alcohol had been taken within $6 \mathrm{~h}$ of the self-harm act were assessed after a median wait of $12 \mathrm{~h}$ (IQR 6-20) compared with $9 \mathrm{~h}$ (IQR 5-19) where no alcohol was involved. Episodes receiving specialist mental health assessment were more likely to result in follow-up care arrangements to specialist mental health outpatient services (45\% vs $13 \%$ for non-assessed episodes, $\mathrm{p}=<0.001)$ or to non-statutory services $(21 \%$ vs $12 \%, \mathrm{p}=<0.001)$. A Wilcoxon signed-rank test showed that the assessment rate was lower among those who self-cut as a method of harm $(\mathrm{z}=-3.745$, $\mathrm{p}=<0.001)$ than those who self-poisoned, with a median hospital rate of $45 \%$ (IQR 28-63\%) vs $58 \%$ (IQR 48$73 \%$ ), respectively. In $15 \%$ of episodes, the individuals 
did not wait or refused assessment, 14\% among episodes involving self-poisoning and $18 \%$ for self-cutting episodes $(\mathrm{p}=0.005)$.

An assessment was conducted in only $68 \%(38 / 56)$ of episodes resulting in admission to an Intensive Care Unit (in one episode, the patient self-discharged prior to assessment) compared with $57 \%$ overall $\left(\chi^{2}=2.66\right.$, $\mathrm{p}=0.10)$. An assessment was conducted in $74 \%(60 / 81)$ of episodes involving strangulation, a higher proportion than overall $\left(\chi^{2}=9.63, p=0.002\right)$, and in $7 \%(6 / 81)$ of episodes the patients did not wait for assessment.

\section{Variation in management of episodes between hospitals}

There was wide variation between the 32 study hospitals in the proportion of episodes in which patients received key aspects of clinical management (table 1 and online supplementary appendix 4 ). The proportion in which a psychosocial assessment was conducted varied from $24 \%$ to $88 \%$. The proportion admitted to a medical ward varied between $22 \%$ and $85 \%$. Each of the 32 hospitals had some form of short-stay ward or observation/assessment unit and medical admission here included referrals to these beds. There was no significant correlation between the proportion of episodes involving poisoning with drugs and the proportion admitted to a medical bed (Spearman's $\mathrm{r}=0.249, \mathrm{p}=0.17$ ). Admission to a psychiatric ward ranged from one hospital where there were no admissions to another where $21 \%$ of episodes resulted in inpatient care. The proportion of episodes resulting in a referral for specialist mental health follow-up (excluding admission to a psychiatric ward) ranged from $11 \%$ to $64 \%$ (median $28 \%$; IQR 22-38\%).

\section{Comparison between 2001-2002 and 2010-2011}

While we could identify repeat episodes by the same individuals in the present study, the 2001/2002 study was solely episode based. To enable like-for-like comparisons with the earlier study, the following analyses are based on all episodes presenting to the 31 hospitals (of the original 32) that were recruited for the current study.

As the duration of data collection for the earlier study and the present study varied, we compared the average number of self-harm attendances per 4-week period in 2001/2002 and 2010/2011. We found an overall 24\% increase in episodes (2075 vs 2563) and a 15\% increase in the median number of episodes per hospital (65 (IQR 42-80) vs 75 (IQR 54-104)). Twenty-five of 31 hospitals had a higher number of episodes in 2010/2011 than in 2001/2002. We compared the overall median proportions of episodes receiving key aspects of clinical management in the two time periods (table 2). The proportion of episodes in which psychosocial assessment occurred was similar, with wide variation in assessment rates between hospitals in both study periods. A higher proportion of episodes in the present study resulted in admission to a medical ward compared with the previous study (an increase of 15\%). The type of general hospital medical ward admitted to differed in the two time periods (2010/2011 vs $2001 / 2002): 32 \%$ vs $56 \%$ were to a general medical bed; $63 \%$ vs $28 \%$ to a short-stay Medical Assessment Unit/Clinical Decision Unit attached to the ED and 5\% vs $16 \%$ other bed (not specified). The median proportion of episodes receiving specialist mental health follow-up (including inpatient admission, referral to outpatient psychiatric care, Crisis Teams, Community Mental Health Teams and statutory drug and alcohol services) decreased by $13 \%$. The median proportion of episodes in which an assessment was conducted with no evidence of subsequent follow-up arrangements (including no general practitioner follow-up) was $3 \%$ compared with $10 \%$ in 2001/2002 ( $\mathrm{p}=0.19$ using a matched-pairs signed-rank test).

\section{Comparison of service provision between 2001-2002 and 2010-2011 (service interviews)}

There was statistical evidence $(p=0.006)$ that service quality, as indexed by our service quality scale, was better in 2010-2011 vs 2001-2002 and the range in scores in the earlier study (threefold) was greater than in the current study (twofold difference). The total Service Scale score had increased in $74 \%(23 / 31)$ of hospitals, decreased in $7 / 31$ hospitals and not changed in one hospital since 2001. The median score had increased from 11.5 to 14.5 in the present study, an increase of $26 \%$.

The individual items which had shown improvement in the greatest number of hospitals were presence of a formal arrangement with Social Services to visit and offer advice to self-harm patients, regular (at least once a year)

Table 1 Summary hospital characteristics and variation in management of self-harm patients across 32 English hospitals in 2010/2011

\begin{tabular}{|c|c|c|c|c|c|c|c|}
\hline & $\begin{array}{l}\text { Service scale } \\
\text { score } \\
\text { (maximum 21) }\end{array}$ & $\begin{array}{l}\text { Total } \\
\text { individuals } \\
\text { during audit }\end{array}$ & $\begin{array}{l}\text { Total } \\
\text { episodes } \\
\text { during audit }\end{array}$ & $\begin{array}{l}\text { Episodes } \\
\text { receiving } \\
\text { specialist } \\
\text { psychosocial } \text { assessment }^{*}\end{array}$ & $\begin{array}{l}\text { Episodes } \\
\text { admitted to } \\
\text { a medical } \\
\text { bed }\end{array}$ & $\begin{array}{l}\text { Episodes } \\
\text { with referral } \\
\text { for mental } \\
\text { health } \\
\text { follow-up } \\
\text { care }^{\star}\end{array}$ & $\begin{array}{l}\text { Episodes } \\
\text { admitted to a } \\
\text { psychiatric } \\
\text { ward* }^{*}\end{array}$ \\
\hline $\begin{array}{l}\text { Median } \\
\text { (range) }\end{array}$ & $14.5(10.5-19)$ & $186(85-450)$ & $223(90-518)$ & $58 \%(24-88)$ & $54 \%(22-85)$ & $28 \%(11-64)$ & $7 \%(0-21)$ \\
\hline
\end{tabular}


Table 2 Changes in service provision and hospital management: 2001-2002 vs 2010-2011 ( $\mathrm{n}=31)$

\begin{tabular}{|c|c|c|c|}
\hline & 2001-2002 & 2010-2011 & p value* \\
\hline Total episodes & 4150 & 7599 & \\
\hline \multicolumn{4}{|c|}{ Specialist mental health assessment $†$} \\
\hline Median, \% (IQR \%) & $55(44-71)$ & $58(45-70)$ & 0.85 \\
\hline \multicolumn{4}{|c|}{ Admission to medical ward } \\
\hline Median, \% (IQR \%) & $39(29-58)$ & $54(41-63)$ & 0.02 \\
\hline \multicolumn{4}{|c|}{ Specialist mental health follow-up† (including admission) } \\
\hline Median, \% (IQR \%) & $51(46-63)$ & $38(26-48)$ & $<0.001$ \\
\hline \multicolumn{4}{|c|}{ Referral to non-statutory mental health/voluntary/other services $\dagger$} \\
\hline Median, \% (IQR \%) & $14(7-20)$ & $15(8-23)$ & 0.24 \\
\hline \multicolumn{4}{|l|}{ Referral to GP† } \\
\hline Median, \% (IQR \%) & $36(22-45)$ & $36(15-64)$ & 0.30 \\
\hline \multicolumn{4}{|l|}{ Psychiatric admission† } \\
\hline Median, \% (IQR \%) & $9(7-15)$ & $7(4-12)$ & 0.05 \\
\hline \multicolumn{4}{|l|}{ Total service scale score } \\
\hline Overall score (\%) & $375.5 / 651(58)$ & $442 / 651(68)$ & \\
\hline Median (IQR) & $11.5(10-14)$ & $14.5(11.5-16)$ & 0.006 \\
\hline
\end{tabular}

service planning/strategy meetings taking place between the specialist mental health and general medical services, and supervision arrangements in place for staff members who undertook psychosocial assessments. Most hospitals (28/31) now had a designated self-harm service (defined as 'any liaison psychiatric service with at least one member of staff located within the ED'), compared with the earlier time period, where this service was available in 23/31 hospitals. Among the 22 hospitals where the Service Scale score had increased (and where the assessment status of the patients was known), 59\% (13/22) had a rate of assessment greater than the median, compared with $25 \%(2 / 8)$ among those with no increase in the Service Scale score $\left(\chi^{2}=2.72, \mathrm{p}=0.099\right)$. Among the seven hospitals whose score had decreased since 2001 (one had remained the same), six no longer had private rooms available in which to carry out the assessment, four no longer allowed all patients admitted to a medical bed to remain in hospital until a psychosocial assessment could be carried out, four no longer routinely provided printed material about local services and four had not audited self-harm services in the past 2 years.

\section{Service score and management}

We found no association between measures of service quality (the total Service Scale score) and the proportion of episodes receiving a specialist psychosocial assessment at each hospital (Spearman's $r=0.141, \mathrm{p}=0.46$ ). There was a positive correlation between the total score and rate of specialist mental health follow-up
(Spearman's $r=0.381, \mathrm{p}=0.038$ ). There was no significant association between a change in score since the previous study and a change in the rate of specialist mental health follow-up (Spearman's $\mathrm{r}=0.171, \mathrm{p}=0.37$ ).

\section{DISCUSSION}

\section{Main findings}

We collected data on over 6400 individuals who had presented with self-harm to 32 general hospitals across England in a 3-month period. The characteristics of our sample were broadly consistent with other hospital-based studies in the UK, ${ }^{12}$ with the majority of episodes related to self-poisoning, and self-harm being more common in younger age groups and women. Alcohol was involved in just over half of the cases and half of the individuals had a previous history of self-harm. There was marked variability in service provision with an approximate 3.5-fold difference between hospitals in the proportion of episodes receiving a specialist assessment, a 4-fold difference in medical admission and an almost 6-fold difference in the proportion of episodes referred for specialist follow-up care. The frequency of admission to a psychiatric ward ranged from one hospital where there were no admissions to another where one in five episodes resulted in inpatient psychiatric care. Overall, 4 of 10 individuals left the hospital without having had an assessment with a mental health specialist.

Disappointingly, given the introduction of clinical guidelines and policy emphasis, the variations in service 
provision were as wide as 10 years previously with no apparent improvement in key aspects of clinical management. Since the earlier study, the proportion of individuals receiving assessment from specialist services had remained static despite the National Institute for Health and Care Excellence (NICE) recommendation that all patients should receive an assessment of risk and needs. Those with more serious methods of harm, such as strangulation, ${ }^{16}$ and those who had been admitted to the Intensive Care Unit had higher rates of assessment than overall (although the difference was not significant in the latter group). This possibly indicated a recognition of the high suicidal intent by clinicians in these groups, although despite their high risk, ${ }^{16}$ assessment was not universal. People who self-cut were less likely than others to be assessed, and yet this group has been shown to be at greater risk of repetition ${ }^{17}$ and future suicide in the UK. ${ }^{18}$ Consistent with other large-scale surveys, ${ }^{19}$ the levels of assessment in those who had cut themselves were reduced-they were less likely to complete treatment and more likely to specifically refuse assessment. The levels of referral for specialist follow-up had decreased, perhaps due to pressures on specialist mental health services with a higher throughput of patients, ${ }^{20}$ problems with accessing specialist services, ${ }^{21}$ or constraints in referral due to the Department of Health recommendation for Community Mental Health Teams to focus care on the severely mentally ill. ${ }^{22}$ The proportion of assessors who were mental health nurses had increased since the earlier study from $46 \%$ to $75 \%{ }^{13}$ but was similar to other current nurse-led self-harm service provision in the UK. ${ }^{23}$ Evidence suggests that psychiatrists and nurses use similar factors to inform their risk assessments, although nurses may be less likely to admit to a psychiatric ward. ${ }^{23}$ This may explain the overall decrease in the proportion of psychiatric admissions since 2001, although the decrease may also reflect trends in the number of psychiatric beds and an increased emphasis on community provision. It should also be noted that nearly one-fifth of the individuals were referred for follow-up to non-statutory services in this study.

The increased proportion of episodes resulting in medical admission may well have reflected the greater use of ED observation and assessment wards rather than an increase in acute admissions to general medical beds. Seventeen hospitals reported that the use of such beds had been introduced or had increased since the earlier study. This increase may partly have been driven by the policy emphasis on reduced waiting times in $\mathrm{EDs}^{24}$ where the target of a maximum of a 4-h stay in the ED before discharge or transfer is recommended. As all hospitals in the current study had short-stay wards or medical observation/assessment units, the variation in proportion of medical admissions between hospitals cannot be attributed to the availability of short-stay wards. Differences between the time of presentation and assessment may be explained by medical fitness and/or intoxication of the patient.
A measure of service quality developed as part of the previous study did show an improvement in 23 of the 31 hospitals with an overall $26 \%$ improvement in the median service quality score. The individual service items that showed an improvement in the greatest number of hospitals related to the availability of supervision, social services input, and joint service planning meetings between mental health and acute care services. Considering changes over time, we found that the overall number of episodes of self-harm in the study centres increased by around one-quarter. However, other studies have found no such increase ${ }^{25}$ and, as we did not correct for changes in population size, our findings might have partly reflected service consolidation rather than a true increase in incidence. We found that 24 of the 25 hospitals with an increased number of self-harm episodes also had an increased number of beds, perhaps suggesting higher levels of activity overall or reflecting hospital mergers.

\section{STRENGTHS AND LIMITATIONS}

This was a large study of hospital attendances to EDs following self-harm using recent data at the individual and episode levels from multiple sites randomly selected from across England. Different electronic systems in the study hospitals required individual methodologies to identify and capture data. However, the robustness of data was affirmed at each study site by the individual data collectors, and pilot data collection was carried out in each centre.

Nevertheless, our findings should be interpreted in the context of a number of methodological limitations. We only collected data on self-harm attendances at hospitals and did not record episodes that did not come to medical attention. As a countrywide descriptive study, the data sources were based on clinical records rather than on in-depth interviews. If there was no information in the notes of an item of interest, then this was coded as absent. As a consequence, some of our findings may underestimate the true prevalence of particular characteristics or associated factors. Another potential weakness was that our data on follow-up were based on referral to services rather than on actual receipt of interventions following discharge from hospital. Although data completeness was high overall and we were able to include 31 of the original 32 sites, there were some difficulties relating to individual hospitals. For example, in one acute Trust, patients received mental healthcare from a variety of mental health providers, so mental health data were unavailable for a small proportion of presentations. Within another site, data sharing agreements between acute and mental health trusts could not be achieved, which again resulted in missing mental health data. The Service Scale measure was developed as part of our previous study and was based on key elements of national guidance. Its use in the current study was principally to allow comparison with data from 2001 
rather than as a stand-alone measure of service quality. Nonetheless, it should be borne in mind that properties of the scale, such as its underlying factor structure, have not been investigated.

\section{Implications for research and practice}

Our study suggests that despite national guidelines and policy initiatives, hospital service provision in England for self-harm patients remains highly variable. This is important because the management patients receive in hospital (particularly the provision of psychosocial assessments) is associated with follow-up care and may well have an impact on outcomes. ${ }^{19}{ }^{26}$ Why have services not shown clear signs of improvement? It is possible, of course, that official guidance has simply not been implemented. Improvements to services may also have been made more difficult due to wider reorganisation of NHS care-increasingly, NHS providers have merged organisations as a response to challenges in delivering care of an acceptable standard within budgetary constraints. ${ }^{27}$ However, there are some indications that services may be getting better in other ways-the number of specialist teams managing self-harm have increased. Composite measures of service quality/provision also seem to have improved: we found higher service scale scores than in the past and these were associated with higher rates of referral to mental health services. This improved quality is not necessarily reflected in all aspects of individual management-on average, only $60 \%$ of individuals receive a psychosocial assessment when they attend hospital following an episode of self-harm and this proportion has remained static over the last decade. This may be partly a result of the increasing demand on services. We should also bear in mind that it is not possible to determine how services would have developed in the absence of guidelines-it is conceivable that the situation would have been much worse than it is currently.

New NICE guidance on the longer term management of self-harm was published in November 2011. ${ }^{28}$ The question of whether this and future policies will have a positive effect on the quality of services and patient outcomes will need careful evaluation. The effect of the new self-harm guideline on future practice may be greater because of the increased focus on implementation (http://guidance.nice.org.uk/CG133) and the development of Quality Standards (key aspects of the quality of care that will be used to commission and assess services in the new NHS). Self-harm Quality Standards were published in June 2013. In terms of future research, developing consistent measures of service quality would be worthwhile. We also need to better understand the link between management and outcome. This is an important but methodologically challenging area and outcomes should include service user evaluation of their experiences. We also need to understand which aspects of treatment are beneficial in routine practice and why, and in which groups of individuals treatments might have the most impact. The role of psychosocial assessment warrants particular attention. ${ }^{19}$ Randomised trials of national service-level interventions are sometimes possible, ${ }^{29}$ and when they are not, observational designs (eg pre-post studies ${ }^{30}$ ) may be worthwhile. Of course, linking findings on variability of services to outcomes is of interest, but this would require substantial further analysis that goes beyond the scope of the current report. However, we hope to address these issues in future publications.

\section{CONCLUSION}

National guidelines and policy initiatives appear to have had little impact on the variability of self-harm service provision. Around $60 \%$ of individuals can expect a psychosocial assessment when they attend hospital following an episode of self-harm, and this proportion has remained static over the last decade or so. There is some evidence to suggest that the overall quality score of self-harm services may have improved, although this is not borne out by individual process measures of hospital management.

Acknowledgements Avon and Wiltshire Mental Health Partnership NHS Trust host the research programme. We would also like to thank the Local Collaborators at each of the 62 NHS Trusts, NHS staff and Clinical Science Officers attached to the Mental Health Research Network who assisted locally in the setting up and data collection for this study.

Contributors NK and $\mathrm{JC}$ designed the study with input from $\mathrm{DG}, \mathrm{OB}, \mathrm{AH}$ and $\mathrm{KH}$. JC was responsible for data collection with assistance from $\mathrm{OB}$ and $\mathrm{ML}$. $\mathrm{JC}$ and SS extracted and processed the data. SS analysed the data with assistance from NK and JC who also interpreted the results. JC wrote the first draft of the paper. All authors contributed to subsequent drafts and have approved the final version of the manuscript.

Funding This paper presents independent research funded by the National Institute for Health Research (NIHR) under its Programme Grants for Applied Research Programme (Grant Reference Number RP-PG-0606-1247). The views expressed are those of the authors and not necessarily those of the NHS, the National Institute for Health Research or the Department of Health.

Competing interests DG, KH and NK are members of the Department of Health's (England) National Suicide Prevention Advisory Group. NK was the chair not only of the NICE guideline development group for the longer term management of self-harm, but also of the NICE Topic Expert Group (which developed the quality standards for self-harm services) and the NICE evidence update for self-harm. KH and DG are the NIHR Senior Investigators. KH is also supported by the Oxford Health NHS Foundation Trust and NK by the Manchester Mental Health and Social Care Trust.

Ethics approval Tameside \& Glossop Research Ethics Committee.

Provenance and peer review Not commissioned; externally peer reviewed. Data sharing statement No additional data are available.

Open Access This is an Open Access article distributed in accordance with the Creative Commons Attribution Non Commercial (CC BY-NC 3.0) license, which permits others to distribute, remix, adapt, build upon this work noncommercially, and license their derivative works on different terms, provided the original work is properly cited and the use is non-commercial.

See: http://creativecommons.org/licenses/by-nc/3.0/

\section{REFERENCES}

1. Bergen $\mathrm{H}$, Hawton $\mathrm{K}$, Waters $\mathrm{K}$, et al. Premature death after self-harm: a multicentre cohort study. Lancet 2012;380:1568-74. 
2. Sinclair JMA, Gray A, Hawton K. Systematic review of resource utilization in the hospital management of deliberate self-harm. Psychol Med 2006;36:1681-93.

3. Taylor TL, Hawton K, Fortune S, et al. Attitudes towards clinical services among people who self-harm: systematic review. $\mathrm{Br} J$ Psychiatry 2009;194:104-10.

4. Hunter C, Chantler K, Kapur N, et al. Service user perspectives on psychosocial assessment following self-harm and its impact on further help-seeking: a qualitative study. J Affect Disord 2013;145:315-23.

5. Blake DR, Mitchell JRA. Self-poisoning: management of patients in Nottingham, 1976. BMJ 1978;1:1032-5.

6. Kapur N, House A, Creed F, et al. Management of deliberate self poisoning in adults in four teaching hospitals: descriptive study. BMJ 1998;316:831-2.

7. Bennewith O, Gunnell D, Peters TJ, et al. Variations in the hospital management of self-harm in adults in England: an observational study. BMJ 2004;328:1108-9.

8. National Institute for Health and Clinical Excellence. The short-term physical and psychological management and secondary prevention of self-harm in primary and secondary care. Clinical Guideline 16. NICE, 2004.

9. Royal College of Psychiatrists. Assessment following self-harm in adults. London: Royal College of Psychiatrists, 2004.

10. Royal College of Psychiatrists. Better services for people who self-harm project. London: Royal College of Psychiatrists, 2005.

11. Dickson S, Steeg S, Gordon M, et al. Self-harm in Manchester 2008-2009. The Centre for Suicide Prevention, The University of Manchester, 2011.

12. Hawton K, Bergen H, Casey D, et al. Self-harm in England: a tale of three cities. Soc Psychiatry Psychiatr Epidemiol 2007;42:513-21.

13. Gunnell D, Bennewith O, Peters TJ, et al. The epidemiology and management of self-harm amongst adults in England. J Public Health 2005;27:67-73.

14. Statacorp. Stata Statistical Software: Release 11. Stata Corporation, College Station, 2009.

15. SPSS Inc. SPSS for Windows Release 19.0.0. Chicago: SPSS Inc, 2010.

16. Runeson B, Tidemalm D, Dahlin M, et al. Method of attempted suicide as predictor of subsequent successful suicide: national long term cohort study. BMJ 2010;340:c3222.
17. Lilley R, Owens D, Horrocks J, et al. Hospital care and repetition following self-harm: a multicentre comparison of self-poisoning and self-injury. Br J Psychiatry 2008;192:440-5.

18. Cooper J, Kapur N, Webb R, et al. Suicide following deliberate self-harm: a 4 year cohort study. Am J Psychiatry 2005;162:297-303

19. Kapur N, Murphy E, Cooper J, et al. Psychosocial assessment following self-harm: results from the multi-centre monitoring of self-harm project. J Affect Disord 2008;106:285-93.

20. King C. Severe mental illness: managing the boundary of a CMHT. $J$ Mental Health 2001;10:75-86.

21. Mind for better mental health. Mental health crisis care: commissioning excellence. A briefing for Clinical Commissioning Groups. November 2012. http://www.mind.org.uk/assets/0002/2011/ CommissioningExcellence_WEB_VERSION_2.pdf

22. Department of Health. Mental health policy implementation guide. London: Community Mental Health Team, The Stationery Office, 2002.

23. Murphy E, Kapur N, Webb R, et al. Risk assessment following self-harm: comparison of mental health nurses and psychiatrists. J Adv Nurs 2011;67:127-39.

24. Department of Health. The NHS Plan. A Plan for Investment. A Plan for Reform London Stationery Office (2000) accessed April 2013. http://webarchive.nationalarchives.gov.uk/20130107105354/http:// www.dh.gov.uk/en/Publicationsandstatistics/Publications/ PublicationsPolicyAndGuidance/DH 4002960

25. Bergen $\mathrm{H}$, Hawton $\mathrm{K}$, Waters $\mathrm{K}$, et al. Epidemiology and trends in non-fatal self-harm in three centres in England: 2000-2007. Br J Psychiatry 2010;197:493-8.

26. Barr W, Leitner M, Thomas J. Self-harm patients who take early discharge from the accident and emergency department: how do they differ from those who stay? Accid Emerg Nurs 2004;12:108-13.

27. Ham C, Dixon A. Tackling the problems of seriously challenged NHS providers. BMJ 2012;344:e4422.

28. National Institute for Health and Clinical Excellence. The long term care and treatment of self-harm. Clinical Guideline 133. NICE, 2011

29. Burns T, Rugkåsa J, Molodynski A, et al. Community treatment orders for patients with psychosis (OCTET): a randomised controlled trial. Lancet 2013;381:1627-33.

30. While D, Bickley $\mathrm{H}$, Roscoe A, et al. Implementation of mental health service recommendations in England and Wales and suicide rates, 1997-2006: a cross-sectional and before-and-after observational study. Lancet 2012;379:1005-12. 